\title{
Phase diagram of the half-filled Hubbard chain with next-nearest-neighbor hopping
}

\author{
S. Daul ${ }^{1,2}$ and R.M. Noack ${ }^{2}$ \\ 1 Physics Department, University of California, \\ Santa-Barbara CA 93106-9530. \\ 2 Institut de Physique Théorique, Université de Fribourg, \\ CH-1700 Fribourg, Switzerland.
}

\begin{abstract}
We investigate the ground-state phase diagram of the half-filled one-dimensional Hubbard model with next-nearest-neighbor hopping using the Density-Matrix Renormalization Group technique as well as an unrestricted Hartree-Fock approximation. We find commensurate and incommensurate disordered magnetic insulating phases and a spin-gapped metallic phase in addition to the onedimensional Heisenberg phase. At large on-site Coulomb repulsion $U$, we make contact with the phase diagram of the frustrated Heisenberg chain, which has spin-gapped phases for sufficiently large frustration. For weak $U$, sufficiently large next-nearest-neighbor hopping $t_{2}$ leads to a band structure with four Fermi points rather than two, producing a spin-gapped metallic phase. As $U$ is increased in this regime, the system undergoes a Mott-Hubbard transition to a frustrated
\end{abstract} antiferromagnetic insulator.

The one-dimensional Hubbard model is the prototypical model for strongly interacting electrons in one dimension. For repulsive interaction, its low-energy, longdistance physics is well-described by the Luttinger liquid picture, in which the fundamental excitations are gapless bosonic spin and charge modes, and the correlation functions exhibit critical behavior with non-universal exponentst. At half filling, Umklapp processes lead to a gap in the charge excitation spectrum and thus insulating behavior for any finite value of the on-site Coulomb interaction, $U$. The spin excitations behave as in the strong-coupling, Heisenberg limit, i.e. are gapless with linear dispersion.

The introduction of a next-nearest-neighbor hopping can change this picture dramatically. In strong coupling, the additional hopping leads to a frustrating next-nearest-neighbor Heisenberg interaction so that the model maps to the frustrated Heisenberg chain. At weakcoupling, the effect of $t_{2}$ is to change the band structure, and, in particular, the number of Fermi points. In this paper, we shall explore the interplay between the frustration at strong coupling and the changed band structure at weak coupling. As we shall see, the resulting phase diagram contains a number of highly interesting phases: a spin-gapped metallic phase, commensurate and incommensurate disordered magnetic insulating phases, as well as the one-dimensional Heisenberg insulator.

We study the Hamiltonian

$$
\begin{aligned}
& H=-t_{1} \sum_{i, \sigma}\left(c_{i+1 \sigma}^{\dagger} c_{i \sigma}+\text { h.c. }\right) \\
& -t_{2} \sum_{i, \sigma}\left(c_{i+2 \sigma}^{\dagger} c_{i \sigma}+\text { h.c. }\right)+U \sum_{i} n_{i \uparrow} n_{i \downarrow},
\end{aligned}
$$

where $t_{1}$ is the nearest-neighbor and $t_{2}$ the next-nearest neighbor hopping and $U$ is the on-site Coulomb repulsion. It is useful to visualize the geometry as a zigzag structure as depicted in Fig. 11.

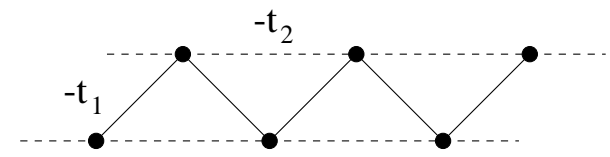

FIG. 1. The $t_{1}-t_{2}$ Hubbard chain.

Here the summation goes over $L$ sites and spin $\sigma$, and we will always take $U$ positive and set $t_{1}=1$. Since the sign of $t_{2}$ is irrelevant at half filling due to particlehole symmetry, we only consider $t_{2}>0$ in the following. For $U=0$ and periodic boundary conditions, $H$ can be diagonalized via a Fourier transform, yielding

$$
H=\sum_{k, \sigma} \varepsilon(k) c_{k \sigma}^{\dagger} c_{k \sigma},
$$

with $k$ an integer multiple of $\frac{2 \pi}{L}$ and

$$
\varepsilon(k)=-2 t_{1} \cos k-2 t_{2} \cos 2 k \text {. }
$$

An interesting feature of this band structure is that there is a nontrivial transition as a function of $t_{2}$ even at $U=0$. For $t_{2}<0.5$, the noninteracting band has two Fermi points and for $t_{2}>0.5$, it has four Fermi points $\left( \pm k_{F}^{(1)}\right.$ and $\left.\pm k_{F}^{(2)}\right)$. This is important in a weak-coupling picture because the Fermi points are separated by the Umklapp vector $q=\pi$ only for $t_{2}<0.5$.

For large $U$, Eq. (11) can be expanded perturbatively in $1 / U$, leading to the one-dimensional frustrated Heisenberg Hamiltonian

$$
H=\sum_{i}\left(J_{1} S_{i} S_{i+1}+J_{2} S_{i} S_{i+2}\right)
$$

with $J_{1}=\frac{4 t_{1}^{2}}{U}$ and $J_{2}=\frac{4 t_{2}^{2}}{U}$. This model has been extensively studied using a number of different methods 2 . In particular, there is an exact solution at $J_{2} / J_{1}=0.5$ $\left(t_{2} / t_{1}=1 / \sqrt{2}\right)$ due to Majumdar and Ghosh 3 . At this point, the ground state is a simple dimer configuration 
and the spectrum has a spin gapt. For $J_{2} / J_{1}<J_{c} \approx$ 0.241167 , stydies combining numerical and field theory calculations 5 have shown that the spin excitation spectrum is gapless. In the strong-coupling expansion, this parameter value maps to the point at which the Fermi surface jumps from two points to four in the $t_{1}-t_{2} \mathrm{Hub}-$ bard chain. When $J_{c}<J_{2} / J_{1} \leq 0.5$, dimerization correlations are present and for $J_{2} / J_{1}>0.5$, i.e. above the Majumdar-Ghosh point3, incommensurate spiral spin correlations appear.

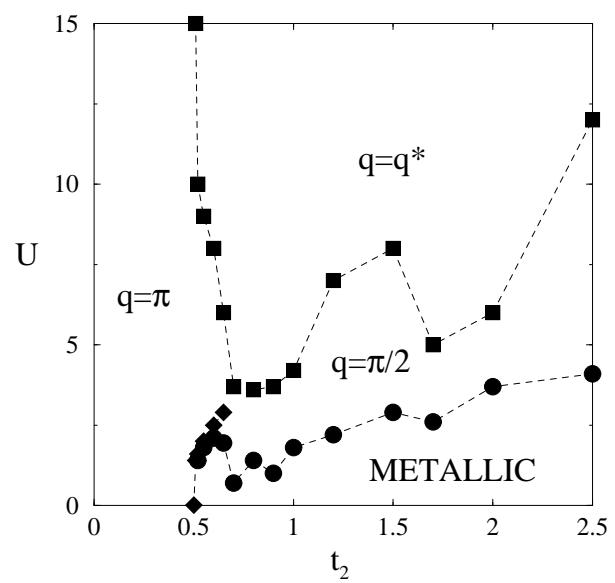

FIG. 2. The unrestricted Hartree-Fock phase diagram. The insulating phases are labelled by the wave vector $q$ of the magnetic ordering for the phases in which the order parameter $m>0$.

Mean-field theory can be a useful tool to provide qualitative information about the ground-state phase diagram even though it tends to overemphasize ordered phases and cannot yield the correct critical behavior. Here we perform mean-field calculations starting from the unrestricted Hartree-Fock Hamiltonian

$$
H_{\mathrm{HF}}=H_{0}+\frac{U}{2} \sum_{\ell}\left(\rho_{\ell} n_{\ell}-\mathbf{m}_{\ell} \cdot \mathbf{s}_{\ell}\right)
$$

where $H_{0}$ is the non-interacting Hamiltonian, $n_{\ell}$ is the total electron density on a site, and $\mathbf{s}_{\ell}=\sum_{s, s^{\prime}} c_{\ell s}^{\dagger} \sigma_{s, s^{\prime}} c_{\ell s^{\prime}}$ with $\sigma_{s, s^{\prime}}^{(x, y)}$ the Pauli matrices. The mean fields $\rho_{\ell}$ and $\mathbf{m}_{\ell}$ are determined by the self-consistent equations $\rho_{\ell}=$ $\left\langle n_{\ell}\right\rangle$ and $\mathbf{m}_{\ell}=\left\langle\mathbf{s}_{\ell}\right\rangle$. We postulate a uniform density $\rho_{\ell}=\rho$, so that $\sum_{\ell} \rho_{\ell} n_{\ell}=\rho N$ is constant, and a spiral arrangement of the magnetic moment

$$
\mathbf{m}_{\ell}=m(\cos q \ell, \sin q \ell, 0) .
$$

The ground-state energy $E_{0}$ is then a function of the variational parameters $q$ and $m$ and the mean-field gap is $\Delta=\frac{U m}{2}$. In order to obtain $q$ and $m$, we minimize $E_{0}(q, m)$ numerically for large systems. When $m=0$, the system is metallic, but if $m$ is finite, the half-filled system is a magnetically ordered insulator with principal wave vector $q$.
We obtain the mean-field phase diagram shown in Fig. 2. For $t_{2}<0.5$, the system is an antiferromagnet (i.e. $q=\pi)$ for all $U>0$. This phase is the same as that obtained for $t_{2}=0$ : The Fermi points are separated by the wave vector $q=\pi$, leading to antiferromagnetic ordering. For $t_{2}>0.5$, this commensurate separation of the Fermi points is absent, and the system is a paramagnetic metal for small $U$.

As $U$ is increased, the system undergoes a transition to an insulating phase with magnetic ordering at wave vector $q=k_{F}^{(2)}-k_{F}^{(1)}=\pi / 2$. For still larger $U$, there is an incommensurate phase with ordering at wave vector $q^{*}$, where $q^{*}$ goes continuously from $\pi$ at smaller $t_{2}$ to $\pi / 2$ as $t_{2} \rightarrow \infty$. As we shall see in the following, the phase boundaries and wave vectors found in this mean-field phase diagram are qualitatively similar to those found for the fully interacting system using the DMRG, although the nature of the phases themselves is different.

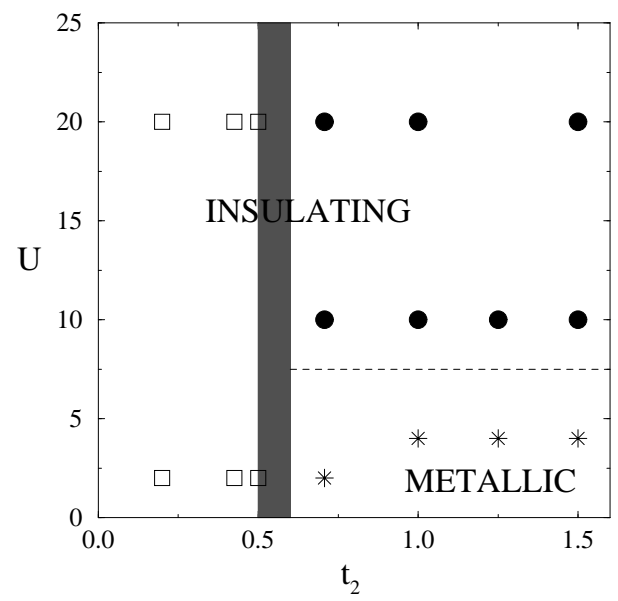

FIG. 3. DMRG phase diagram at half filling. The symbol $\square$ indicates a C0S1 phase, $\bullet$ a C0S0 phase and $*$ a C1S0 phase. The approximate phase boundaries are marked as a guide to the eye.

Using the DMRG6, we can obtain the ground-state energy and wave function on a finite lattice numerically to very high accuracy. Here we perform calculations keeping up to 800 states on lattices of up to 64 sites so that the maximum weight of the discarded density matrix eigenvalues is $10^{-6}$. In order to determine the phase diagram, we first calculate the charge and spin gaps, defined as

$$
\begin{aligned}
\Delta_{\rho} & =\frac{1}{2}\left[E_{0}(N+2,0)+E_{0}(N-2,0)-2 E_{0}(N, 0)\right] \\
\Delta_{\sigma} & =E_{0}(N, 1)-E_{0}(N, 0)
\end{aligned}
$$

where $E_{0}(N, S)$ is the ground-state energy for $N$ particles and spin $S$. We calculate the excitation gaps for systems with different size $L$ and then extrapolate to $L \rightarrow \infty$ using a quadratic polynomial in $\frac{1}{L}$. We have calculated the spin gap as a function of $U$ in the large $U$ limit (e.g. at $U=100$ ) and find very good quantita- 
tive agreement with numerical results for the frustrated Heisenberg chain from Ref.2.

In Fig. 3, we display the phase diagram in the $U-t_{2}$ plane determined using the $L \rightarrow \infty$ spin and charge gaps computed with the DMRG. We label the phases using the notation of Ref. 母, in which $\mathrm{C} n \mathrm{~S} m$ represents a phase with $n$ gapless charge modes and $m$ gapless spin modes. For $t_{2}<0.5$, the system is in the one-dimensional Heisenberg phase found for $t_{2}=0$, a C0S1 phase. As in the mean-field phase diagram, this phase extends to the entire region in which there are two Fermi points. For $t_{2}>0.5$, there are two different regions both with gapped spin modes. For weak $U$, the system is metallic (C1S0), and for $U>U_{c}$ of the order of the bandwidth, the system is insulating $(\mathrm{COS} 0)$. In this region, where the system has four Fermi points, a weak-coupling renormalization group calfulation predicts a metallic spin liquid i.e. C1S0, phase, Such a spin-gapped phase has been found numerically for this model away from half filling At half filling, there are no relevant Umklapp processes which could drive the system to an insulating phase when there are four Fermi points.

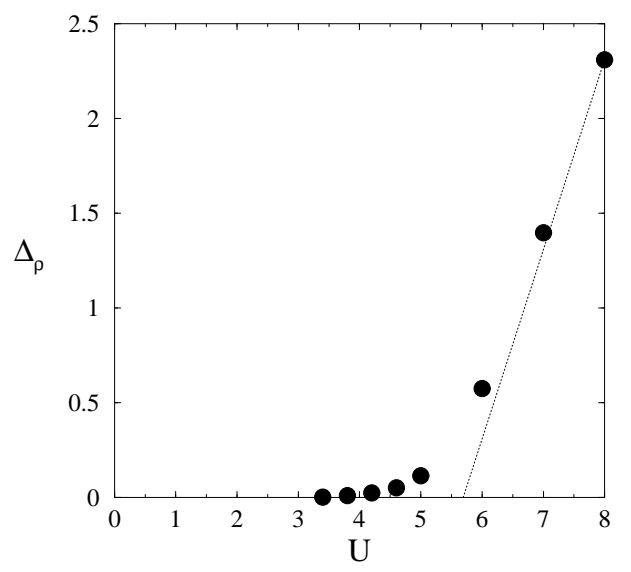

FIG. 4. Charge gap as a function of $U$ for $t_{2}=1$ at half filling. The dotted line is linear in $U$.

In Fig. A, we show the charge gap as a function of $U$ for $t_{2}=1$. There is a clearly defined metal-insulator transition at $U_{c}=3.2 \approx W / 2$, where $W$ is the bandwidth of the non-interacting case $\left(W=6.25\right.$ for $\left.t_{2}=1\right)$. For $U \gg U_{c}$, the gap grows linearly as $U$ - J as one would expect above a Mott-Hubbard transition 10 , but the curve is rounded near the transition point. The metal-insulator transition as a function of $U$ therefore appears to be continuous.
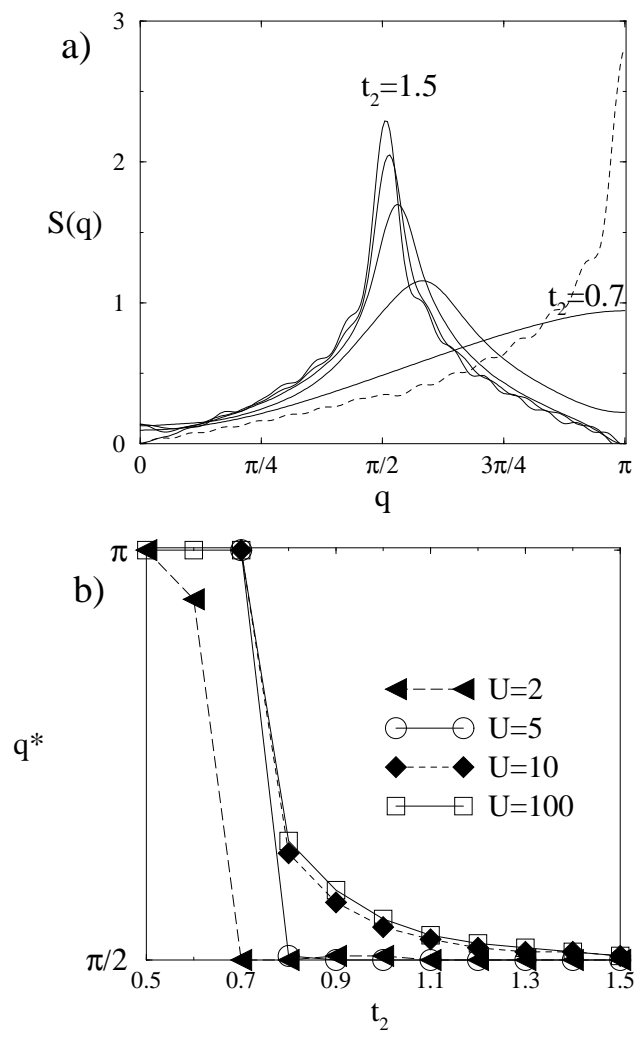

FIG. 5. (a) Fourier transform, $S(q)$, of the spin-spin correlation function for the half-filled system with $L=40, U=100$ and $t_{2}=0.7 \ldots 1.5$ in steps of 0.2 (solid lines from bottom to top at $q=\pi / 2$ ); the dashed line is the result for $t_{2}=0$. (b) The wavevector $q^{*}$ obtained from the position of the peak in $S(q)$ as a function of $t_{2}$ for various values of $U$.

We now investigate the nature of the magnetic ordering in the different phases by examining the spin-spin correlation function

$$
S_{\mathrm{av}}(r)=\sum_{\{\ell\}}\left\langle s_{\ell}^{+} s_{\ell+r}^{-}\right\rangle
$$

where $s_{\ell}^{+}\left(s_{\ell}^{-}\right)$are the spin raising (lowering) operators corresponding to $\mathbf{s}_{\ell}$, and we average over a number of $\ell$-values (typically six) to reduce oscillations present because of the open boundaries. We then perform a continuous Fourier transform to obtain the static structure factor

$$
S(q)=\int_{-\infty}^{\infty} d r e^{i q r} S_{\mathrm{av}}(r)
$$

The resulting function is plotted in Fig. 5(a) for various $t_{2}$ ranging from 0.7 to 1.5 . It is clear that the peak shifts continuously from $q=\pi$ at $t_{2}=0.7$ to $q=\pi / 2$ at $t_{2}=1.5$. As $t_{2}$ increases, the peak at $q=\pi / 2$ becomes sharper, resembling more closely the $q=\pi$ peak of $t_{2}=0$ structure factor, also shown for reference. This is because the correlation length diverges as $t_{2}$ becomes large 2 ; it is the limit of two uncoupled Heisenberg chains. 
The position of the peak, $q^{*}$, is plotted as a function of $t_{2}$ in Fig. 5(b) for different $U$ values. For $U \geq 5$, $q^{*}=\pi$ for $t_{2}<t_{2}^{*} \approx 0.7$. At large $U$, one expects $q^{*}$ to deviate from $\pi$ only for $t_{2}$ above the Majumdar-Ghosh point, $t_{2}^{\mathrm{MG}}=1 / \sqrt{22}$. As can be seen, $t_{2}^{*} \approx t_{2}^{\mathrm{MG}}$ down to small values of $U$. Note that $t_{2}^{*}$ does not coincide with the opening of the spin gap in Fig. 3; there is an intermediate region of a spin-gapped dimer phase with $q^{*}=\pi$. As $U$ is reduced, the region of incommensurate spiral spin order becomes narrower. This is expected because there is no incommensurate spiral spin order at $U=0$.

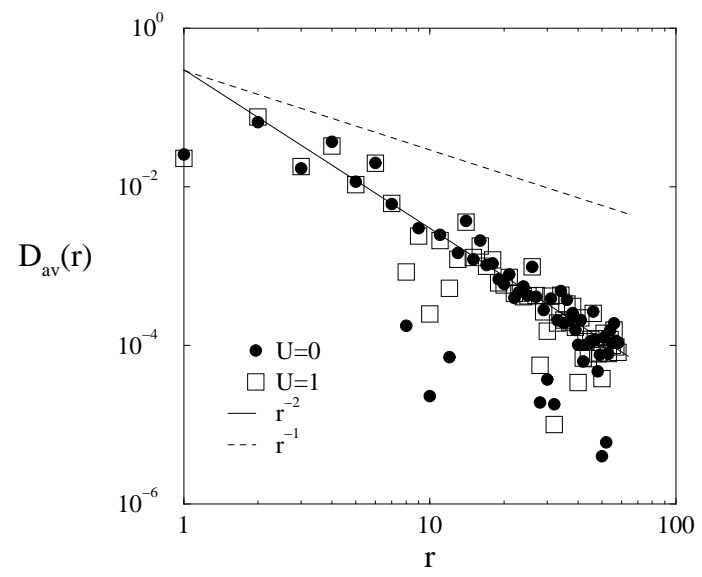

FIG. 6. Pairing correlation function on a log-log scale for $L=64, t_{2}=0.78$ and $U=0,1$.

In the metallic spin-liquid (C1S0) region, the longdistance behavior is characterized by the correlation functions associated with the charge degrees of freedom. A bosonization treatment valid for weak $U$ predicts that two competing correlation functions have the slowest asymptotic decay: the dimer-wave correlation function

$$
\chi_{D W}(x) \sim \frac{\cos \pi x}{x^{\theta}}
$$

and the singlet superconducting correlation function

$$
\chi_{S C}(x) \sim \frac{1}{x^{1 / \theta}} .
$$

If $\theta>1$, the pairing is dominant, while for $\theta<1$ the dimer wave is dominant. In addition, at half filling highorder Umklapp process are relevant for $\theta<1$ and the system becomes insulating 8 . We then expect that $d$-wave pairing correlation are dominant for $U<U_{c}$, where the charge gap opens at $U_{c}$. We compare the pairing function for the weakly interacting and the non-interacting case to see if there is an enhancement. At half filling $k_{F}^{(2)}-k_{F}^{(1)}=$ $\pi / 2$, so the relevant pair operators in real space involve pairs on next-nearest-neighbor sites. We have carefully evaluated the pairing correlation function

$$
D_{\mathrm{av}}(r)=\sum_{\{i\}}\left\langle\Delta_{i} \Delta_{i+r}^{+}\right\rangle
$$

where $\Delta_{i}=\left(c_{i \uparrow} c_{i+2 \downarrow}-c_{i \downarrow} c_{i+2 \uparrow}\right) / \sqrt{2}$ and we average over a number of $i$-values to reduce oscillations present because of the open boundaries. In Fig. 6, we plot the results for $U=0$ and $U=1$, and we clearly see that there is no enhancement when we turn $U$ on. This is in contradiction with previous work by Kuroki et al.11 who claim that the superconducting correlations are dominant for small $U$ based on projector QMC. We have also evaluated the dimer-wave correlation function which shows no enhancement for $U<U_{c}$, but the long range behavior clearly changes from $r^{-2}$ to $r^{-1}$ when $U>U_{c}$.

In conclusion, we have explored the rich ground-state phase diagram of the half-filled $t_{1}-t_{2}$ Hubbard chain using the Density Matrix Renormalization Group. For $t_{2}<0.5$ the model is insulating for all values of $U$ due to Umklapp processes and there is no spin gap. For $t_{2}>0.5$ the system has a spin gap for all $U$ but is metallic (i.e. the charge gap vanishes) for sufficiently small $U$. A continuous metal to insulator transition occurs when $U$ is of the order of the bandwidth. At large $U$, we find quantitative agreement with the phase diagram of the frustrated Heisenberg chain. While the overall phase diagram is in agreement with weak-coupling renormalization group calculations 8 , the pairing correlations do not behave as predicted in the spin-gapped metallic phase. The exact nature of the metallic phase is difficult to determine; more work must be done in this direction.

This work was supported by the Swiss National Foundation under Grant Nos. 20-46918.96 and 20-53800.98. We would like to thank D. Baeriswyl, F. Gebhard and D.J. Scalapino for helpful discussions.

${ }^{1}$ For a recent review, see J. Voit, Rep. Prog. Phys. 58, 9771116 (1995).

${ }^{2}$ S. White and I. Affleck, Phys. Rev. B 54, 9862 (1996); and references therein.

${ }^{3}$ C. K. Majumdar and D. K. Ghosh, J. Math. Phys. 10, 1388 (1969).

${ }^{4}$ I. Affleck, T. Kennedy, E. Lieb and H. Tasaki, Comm. Math. Phys. 115, 477 (1988).

${ }^{5}$ S. Eggert, Phys. Rev. B 54, R9612 (1996).

${ }^{6}$ S. R. White, Phys. Rev. Lett. 69, 2863 (1992); Phys. Rev. B 48, 10345 (1993).

${ }^{7}$ L. Balents and M. P. A. Fischer, Phys. Rev. B 53, 12133 (1996).

${ }^{8}$ M. Fabrizio, Phys. Rev. B 54, 10054 (1996).

${ }^{9}$ S. Daul and R. M. Noack, Phys. Rev. B 58, 2635 (1998).

${ }^{10}$ N. F. Mott, Metal-Insulator Transitions, 2nd ed. (Taylor and Francis, London, 1990); F. Gebhard, The Mott MetalInsulator Transition, Springer Tracts in Modern Physics Vol. 137 (Springer, Berlin, 1997).

${ }^{11}$ K. Kuroki, R. Arita and H. Aoki, J. Phys. Soc. Jap. 66, 3371 (1997). 